\title{
Caracterización tipológica del espacio edificado de las casas de corredor. El caso de las ciudadelas del casco histórico de La Habana y las corralas madrileñas
}

\section{Typological characterization of built space of corridor houses. The case of "ciudadelas" of the historic town in Havana and the corridor houses in Madrid}

R. Fernández Martín $^{(*)}$, J. Santa Cruz Astorqui ${ }^{(*)}$, S. Arbaiza Blanco-Soler ${ }^{(*)}$, M. del Río Merino ${ }^{(*)}$

\section{RESUMEN}

En este artículo se presentan algunos resultados de un proyecto de investigación que pretende la caracterización de los diferentes modelos que en España e Hispanoamérica evolucionaron de las casas de corredor para poder diseñar soluciones válidas que permitan la adaptación de los mismos a los criterios actuales de habitabilidad. En concreto se presentan los resultados de la caracterización en base a: periodo de construcción; geometría y forma de los edificios y geometría del patio, de 286 corralas madrileñas del barrio de Embajadores y de 213 ciudadelas del Centro Histórico de La Habana.

Como consecuencia del estudio se han encontrado grandes coincidencias en ambos modelos, por lo que se concluye que se trata un modelo tipológico característico de los barrios populares antiguos con una presencia tan alta que resulta imposible explicar la trama urbana sin considerar su papel de «relleno» de las manzanas.

Palabras clave: Ciudadelas; casas de corredor; caracterización tipológica; invariantes tipológicos.

\section{ABSTRACT}

In this article, some of the results obtained from an investigation aiming to characterize the different models that in Spain and Spanish America developed from original "corridor houses" are presented. This research aims to design valid solutions allowing the adaptation of these houses to habitability current criteria. Specifically, results obtained from the characterization resulting from: date of building, dimensions and shape, and type of the yard and its relationship with corridors in 286 "corridor houses" in Embajadores neighborhood, in Madrid, and 213 so called "ciudadelas" inside the Historic Centre in Havana, are presented.

As a conclusion, great coincidences have been found in both of the models. So that, it can be said that this is about a typological model, characteristic of ancient and popular neighborhoods, with such a high presence that it turns out impossible to explain the urban weave without considering their fundamental role in "filling" blocks.

Keywords: Ciudadelas; corridor houses; typological characterization; typological invariants.

(*) Escuela Técnica Superior de Edificación - Universidad Politécnica de Madrid (España)

Persona de contacto/Corresponding author: rafael.fernandezm@upm.es (R. Fernández Martín)

Cómo citar este artículo/Citation: Fernández Martín, R., Santa Cruz Astorqui, J., Arbaiza Blanco-Soler, del Río Merino, M. (2016). Caracterización tipológica del espacio edificado de las casas de corredor. El caso de las ciudadelas del casco histórico de La Habana y las corralas madrileñas. Informes de la Construcción, 68(541): e135, doi: http://dx.doi.org/10.3989/ic.15.036.

Licencia / License: Salvo indicación contraria, todos los contenidos de la edición electrónica de Informes de la Construcción se distribuyen bajo una licencia de uso y distribución Creative Commons Reconocimiento no Comercial 3.o. España (cc-by-nc). 


\section{INTRODUCCIÓN}

La casa de corredor (en sus diferentes formas y denominaciones) es el resultado de la adaptación y evolución de diferentes modelos arquitectónicos y sociales provenientes de las distintas culturas que confluyeron en la península ibérica a lo largo de su historia y que se adaptaron a las necesidades y circunstancias que se dieron en diferentes lugares, culturas y épocas.

Todos estos modelos tienen una raíz primigenia común en la casa-patio original de las culturas mediterráneas clásicas (la casa-patio griega y posteriormente, la villa romana), y anteriormente en la casa-patio de la antigua Mesopotamia (Figura 1).

La evolución de la casa-patio fue sustancialmente diferente en la cultura cristiana y la musulmana, adquiriendo características propias de cada cultura tanto en la propia concepción formal y funcional de la casa como en la de la ciudad.

El modelo de casa-patio romana se difundió en toda la meseta norte, utilizándose en la repoblación que siguió a la reconquista, pero fusionándose con la concepción de vivienda y ciudad musulmana que, en distinto grado se había materializado ya en la mayor parte de pueblos y ciudades de España. Encontramos así dos modelos de vivienda que están claramente diferenciados: la casa rural castellana, que conserva la tradición visigoda, y la casa musulmana andaluza, como adaptación de los modelos de vivienda de la arquitectura originaria musulmana a la simbiosis de culturas propias del sur de la península.

Es precisamente el modelo procedente de esta fusión el que se exporta al continente americano.

Son varias las características principales que perdurarán de los dos modelos que se fusionan:
De la casa patio castellana (Figura 2), el aspecto formal y organizativo de la configuración de los corredores mediante entramados de madera (1), y el funcional, adoptando dicho patio como representación del espacio público de la plaza (en contraposición al carácter privado del patio musulmán).

De la herencia musulmana queda patente la configuración cerrada de la trama urbana, donde la casa se cierra al exterior, y sin embargo vuelca al interior toda la expresión cultural de las gentes que la habitan (fiestas, celebraciones, ornamentación), dotando a éste modelo de una gran capacidad de supervivencia en el entorno agresivo que suponían las ciudades medievales (2).

Así pues, las casas de corredor resultan de una perfecta simbiosis entre la estructura y forma del modelo de casa patio clásica, y el proceso de agrupación comunal en torno al espacio común (y privado) que representa el patio.

La casa de corredor ha tenido un gran éxito en todas las culturas, en las que siempre ha existido un profundo sentimiento de protección de la intimidad familiar (lo privado) frente a la calle y la ciudad (lo público), sobre todo cuando el entorno próximo de la casa es potencialmente agresivo, o cuando el entorno socio económico propicia su adopción como modelo residencial popular.

Así, aparece este modelo con denominaciones diferentes pero con una morfología similar: en Madrid o en Sevilla, denominándose corralas (Figura 3); en Málaga, denominándose corralones; ciudadelas en Gijón (3) o Santa Cruz de Tenerife y casas de pasillo en Barcelona; pero también ciudadelas en La Habana, Bogotá o San salvador; conventillos en Buenos Aires, Santiago de Chile o Montevideo; cuarterías en San José, Managua o Santo Domingo, inquilinatos en Caracas o Quito, $\mathrm{y}$ vecindades en Mexico D.F.

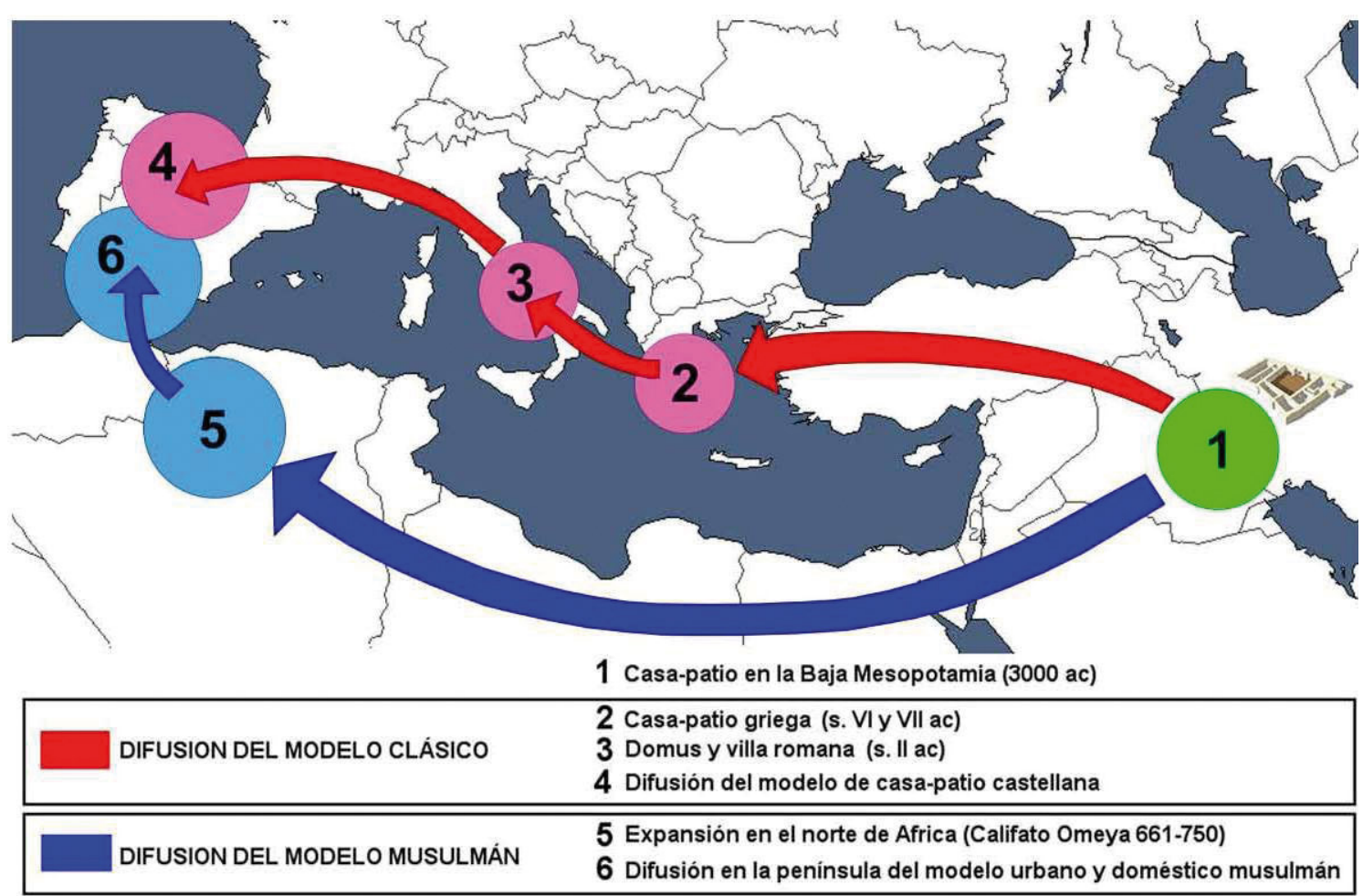

Figura 1. Difusión de los modelos clásico y musulmán de las casas-patio (1). 


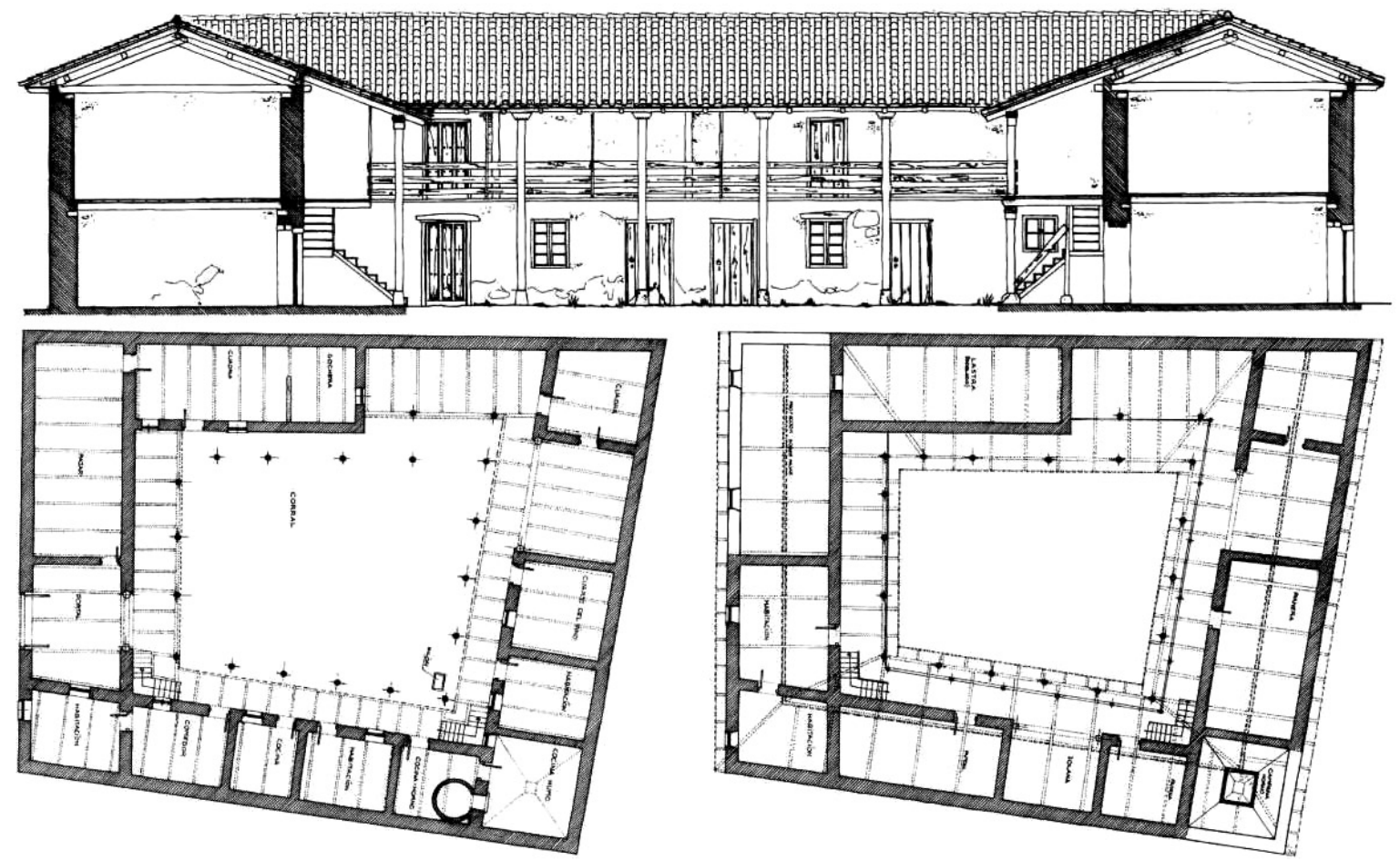

Figura 2. Plantas y sección de una casa de corral (3).

En este artículo se presentan algunos de los resultados de un proyecto de investigación que pretende la caracterización de los diferentes modelos que en España e Hispanoamérica evolucionaron de las casas de corredor para poder diseñar soluciones válidas que permitan la adaptación de los mismos a los criterios actuales de habitabilidad. En concreto, en el proyecto de investigación expuesto en este trabajo, hasta este momento, se ha documentado de manera precisa y exhaustiva la casa de corredor madrileña y la ciudadela de La Habana (Figura 4), llevando a cabo su caracterización morfológica y tipológica, y estableciendo posteriormente una comparativa entre los dos modelos.

\section{METODOLOGÍA}

Para dar respuesta a los objetivos planteados fue preciso inventariar una muestra suficiente de edificios que respondiesen a esta tipología, tanto en Madrid como en La Habana.
En primer lugar se delimitó para ambos casos un área geográfica que permitiese asumir el trabajo de toma de datos, sin perder por ello la representatividad de la muestra.

Una vez delimitadas las zonas de actuación, se llevó a cabo la caracterización de los edificios atendiendo al periodo de construcción, a las relaciones existentes entre su organización espacial y el tamaño y forma del solar en el que se ubica, y a las relaciones geométrico-funcionales existentes ente patio y los corredores.

Los datos utilizados proceden de la información generada en el «Estudio tipológico, constructivo y estructural de las casas de corredor en Madrid» (1), en «Inventario morfológico de las ciudadelas del Casco Histórico de La Habana para su caracterización como tipología específica. Estudio comparado con las corralas madrileñas» (5) y en el «Estudio urbanístico y constructivo de las casas de corredor en Madrid. Investigación y
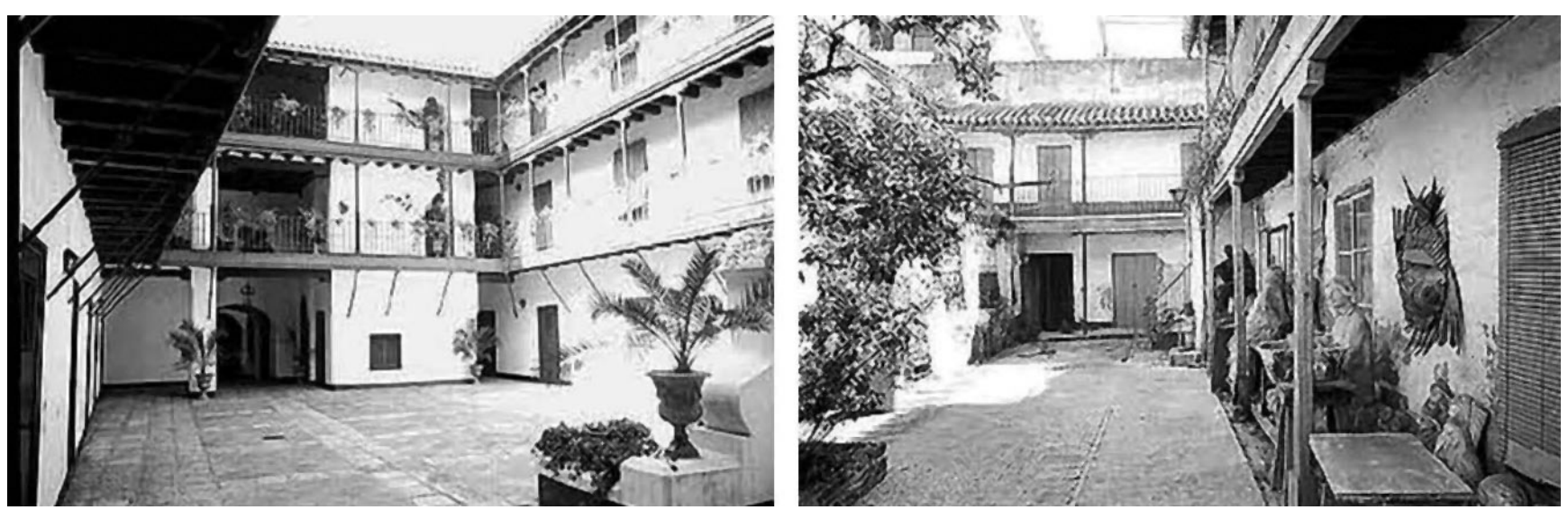

Figura 3. Corral del Conde, del s. XVI (Sevilla) (4). 

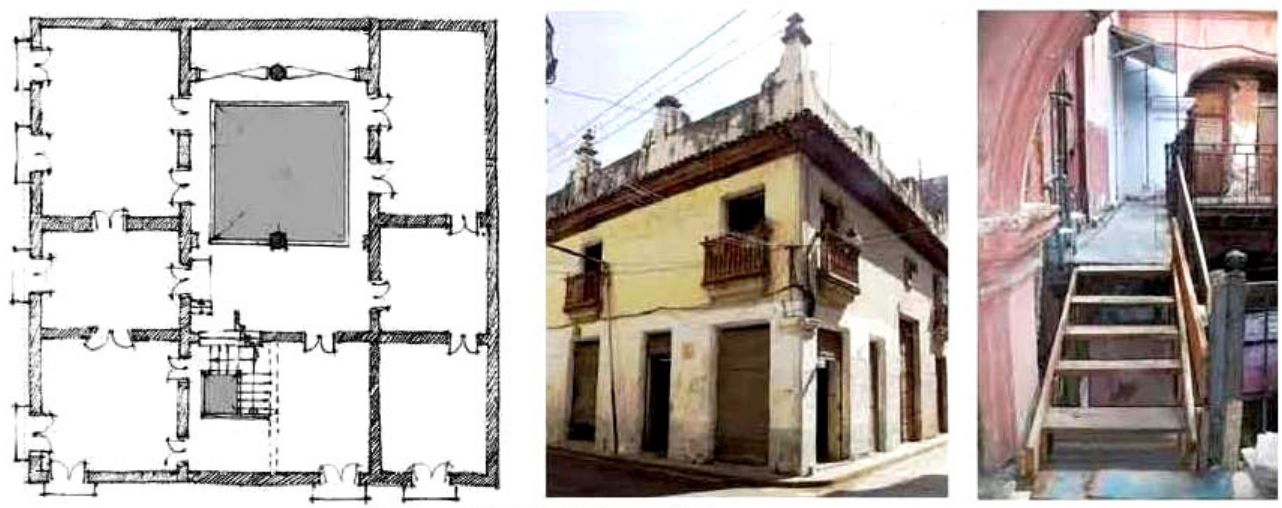

Habana, 901. La Habana Vieja
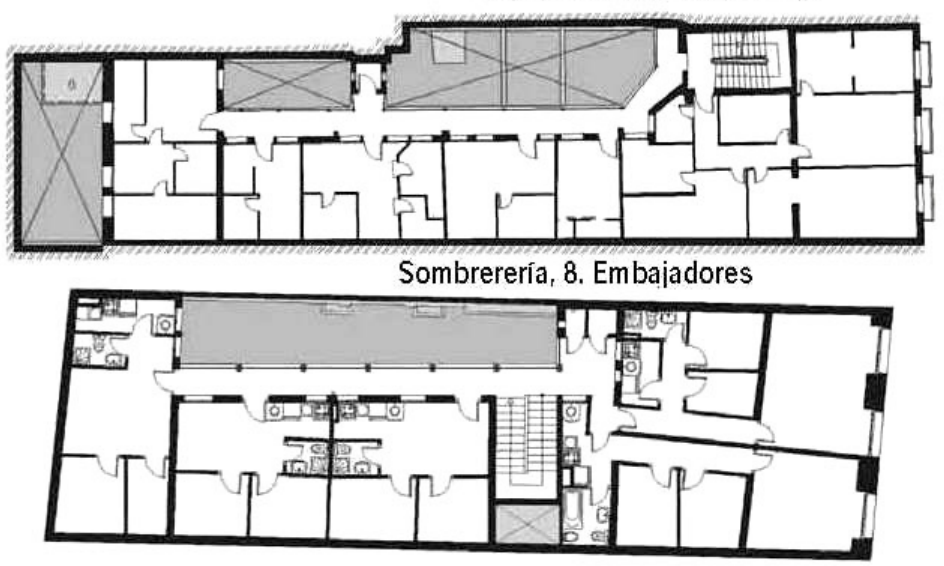

Rodas, 5. Embajadores

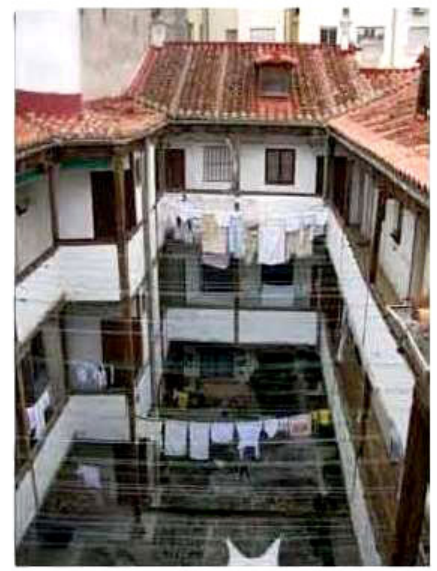

Buenavista, 16. Embajadores

Figura 4. Ciudadelas habaneras y corralas madrileñas.

documentación de las tipologías edificatorias y constructivas de las denominadas casas de corredor o corralas en la Ciudad de Madrid a partir de una relación de edificios proporcionada por la Empresa Municipal de la Vivienda (EMV)» (6).

Para ello, se cumplimentó una ficha de inventario, cuyos datos se trasladaron a una base de datos excel para su posterior análisis. De entre todos ellos, en el presente artículo se presentan resultados referentes a:

- Fecha de construcción del edificio.

- Ubicación geográfica del edificio.

- Superficies del solar, del patio, edificada, etc.

- Datos geométricos y morfológicos del edificio.

Por último, con los datos obtenidos se realizó una comparativa entre los dos modelos (Madrid-La Habana) mediante el programa STATISTICAL PACKAGE FOR THE SOCIAL SCIENCIES (IBM SPSS STATISTIC 21.0) (7) para demostrar la correspondencia existente entre la organización espacial de este tipo de edificios y el tamaño y forma del solar en el que se ubican, así como para buscar relaciones de afinidad entre ellos.

\section{RESULTADOS}

En Madrid, se estudiaron, en el primer trabajo (6) 286 corralas madrileñas situadas en el Distrito Centro,, con un total de 4.293 viviendas (unas 15 viviendas por edificio). En el segundo (1), referido más concretamente al barrio de Embajadores, se registraron 86 corralas, con 2.399 viviendas (28 por edificio).
En el caso de La Habana, se trabajó sobre la base de varios inventarios especializados, realizados entre 1979 y 2010, para la identificación de las ciudadelas (8) (9) (10) inventariándose en el Centro Histórico un total de 213 edificios que responden a la tipología estudiada, con un total de 3.238 viviendas.

Los resultados globales en las ciudadelas habaneras y en las corralas madrileñas estudiadas son los que se muestran en las Tablas 1 y 2.

No obstante, para profundizar en el estudio, en adelante se acotó su ámbito, reduciéndolo al barrio de Embajadores, que contiene el $60 \%$ de las corralas del distrito Centro y cuyas características sociológicas y superficie se acercan más a las de La Habana Vieja (Tabla 3).

De la mera comparación de estos datos se puede concluir que, para un tamaño de solar y una ocupación media semejantes en ambos casos, el número medio de viviendas en las ciudadelas habaneras representa el 54,74 \% y la superficie media construida el 39,27 \% respectivamente de los totales de las corralas madrileñas. Es decir, la densidad de población usuaria de esta tipología edificatoria es algo más del doble en el caso madrileño que en La Habana Vieja, lo que se explica por la diferencia de alturas edificadas, como se verá más adelante.

\section{Caracterización por periodo de construcción}

Atendiendo al periodo de construcción, las ciudadelas que aún persisten en La Habana Vieja se edificaron, en su gran 
Tabla 1. Datos generales de las ciudadelas habaneras.

\begin{tabular}{|l|l|l|}
\hline Superficie de La Habana Vieja & $142,51 \mathrm{Ha}$ & \\
\cline { 1 - 2 } $\mathrm{N}^{0}$ de edificios registrados & 213 & Sup. Media solar: 311,01 \\
\hline Total superficie de solares & $66.243,76 \mathrm{~m}^{2}$ & $\begin{array}{l}\text { Ocupación media: } 83,65 \% \\
\text { Sup. Media S/R: } 586,49 \mathrm{~m}^{2}\end{array}$ \\
\hline Total superficie de patios & $9.798,59 \mathrm{~m}^{2}$ & $\begin{array}{l}\text { 845 exteriores + 2.401 interiores } \\
\text { Sup. construida media edificio: } 587,65 \mathrm{~m}^{2}\end{array}$ \\
\hline Total superficie edificada S/R & $124.923,72 \mathrm{~m}^{2}$ & \\
\cline { 1 - 2 } $\mathrm{N}^{0}$ total de viviendas & 3.246 & \multicolumn{2}{|c|}{} \\
\cline { 1 - 2 } Superficie cons. tot. de viviendas & $125.168,94 \mathrm{~m}^{2}$ & \\
\hline
\end{tabular}

Tabla 2. Datos generales de las corralas madrileñas.

\begin{tabular}{|c|c|c|}
\hline Superficie Distrito Centro & $523,08 \mathrm{Ha}$ & \multirow{7}{*}{$\begin{array}{l}\text { Sup. Media solar: } 360,50 \\
\text { Ocupación media: } 81,80 \% \\
\text { Sup. Media S/R: } 1.310,79 \mathrm{~m}^{2} \\
\text { 2.393 exteriores }+4.660 \text { interiores } \\
\text { Sup. construida media edificio: } 997 \mathrm{~m}^{2}\end{array}$} \\
\hline $\mathrm{N}^{\mathrm{o}}$ de edificios registrados & 286 & \\
\hline Total superficie de solares & $103.120 \mathrm{~m}^{2}$ & \\
\hline Total superficie de patios & $18.770 \mathrm{~m}^{2}$ & \\
\hline Total superficie edificada S/R & $374.914 \mathrm{~m}^{2}$ & \\
\hline $\mathrm{N}^{0}$ total de viviendas & 7.053 & \\
\hline Superficie cons. tot. de viviendas & $285.231 \mathrm{~m}^{2}$ & \\
\hline
\end{tabular}

Tabla 3. Datos sobre superficies y grado de acupación.

\begin{tabular}{|l|r|r|r|r|}
\cline { 2 - 5 } \multicolumn{1}{c|}{} & \multicolumn{2}{c|}{ Habana Vieja } & \multicolumn{2}{c|}{ Embajadores } \\
\cline { 2 - 5 } \multicolumn{1}{c|}{} & \multicolumn{1}{c|}{ Total } & Media (/213) & \multicolumn{1}{c|}{ Total } & \multicolumn{1}{c|}{ Media (/86) } \\
\hline Sup. solar & $66.227,1 \mathrm{~m}^{2}$ & $310,9 \mathrm{~m}^{2}$ & $31.255,3 \mathrm{~m}^{2}$ & $360,4 \mathrm{~m}^{2}$ \\
\hline Ocupación media & & $82,2 \%$ & & $82,3 \%$ \\
\hline Sup. patio & $9.902,7 \mathrm{~m}^{2}$ & $46,5 \mathrm{~m}^{2}$ & $5.665,7 \mathrm{~m}^{2}$ & $65,9 \mathrm{~m}^{2}$ \\
\hline Sup. Edif. S/R & $126.602,9 \mathrm{~m}^{2}$ & $594,4 \mathrm{~m}^{2}$ & $36.921,1 \mathrm{~m}^{2}$ & $429,3 \mathrm{~m}^{2}$ \\
\hline $\mathrm{N}^{\text {O total Viv. }}$ & 3.238 & 15,2 & 2.388 & 27,8 \\
\hline Sup.Constr. viviendas & $84.871,6 \mathrm{~m}^{2}$ & $398,5 \mathrm{~m}^{2}$ & $87.269,1 \mathrm{~m}^{2}$ & $1.014,8 \mathrm{~m}^{2}$ \\
\hline Sup. Media de vivienda & & $26,2 \mathrm{~m}^{2}$ & & $36,6 \mathrm{~m}^{2}$ \\
\hline
\end{tabular}

mayoría, entre 1825 y 1938 , dato que coincide sensiblemente con el obtenido en los estudios de referencia sobre las corralas, tal y como se puede constatar en la Figura 5.

En el plano actual del La Habana Vieja con la situación de las ciudadelas estudiadas grafiadas según su periodo de construcción, se observa una distribución bastante homogénea sin una distinción clara por años de construcción, conclusión lógica si se tiene en cuenta que la fisonomía actual de la trama urbana del casco histórico quedó prácticamente consolidada a finales del siglo XVII.

En lo que respecta a las corralas del madrileño barrio de Embajadores, la distribución de edificios construidos en diferentes épocas es igualmente bastante homogénea, aunque se concentran sobre todo en la franja que se edificó originariamente durante la segunda mitad del s. XVI y, en menor medida, en la franja correspondiente a la ocupación entre 1600 y 1700.

No obstante, no puede olvidarse el hecho de que la consolidación completa del barrio de Embajadores se produce en torno a 180o, y que es allí donde se ubican la mayor parte de las corralas madrileñas. Ello parece indicar que seguramente las corralas no fuesen edificios de nueva planta, sino producto de ampliaciones sucesivas de casas de corredor anteriores de menor altura (casas con huerto trasero típicas de los arrabales) (1).
Por todo ello, y teniendo en cuenta la antigüedad de ambos tipos de casas de corredor, se puede afirmar que en la mayoría de los casos se trata bien de reedificaciones o bien de ampliaciones, sobre antiguos edificios existentes, tanto en altura como en fondo edificado (1). También en ambos casos se puede observar el intenso proceso de renovación al que fueron sometidos los dos barrios entre 1875 y 1925 .

En ambos casos, la mayoría de los edificios cuentan ya con una antigüedad de entre 110 y 150 años, lo que en el precario caso habanero perjudica aún más su estado de conservación.

\section{Caracterización por superficie y grado de ocupación del solar}

Se muestran a continuación los gráficos porcentuales comparativos de ambos tipos de edificaciones relativos a su superficie, grado de ocupación, superficie edificada sobre rasante y al número de plantas tanto en fachada a calle como a patio.

En la Figura 6, se puede comprobar la homogeneidad en la extensión de los solares en los dos modelos, tanto en el tamaño propiamente dicho como en el rango más frecuente de superficie. Las superficies de solar, es decir, las definidas por el perímetro de la propiedad, se han obtenido, en ambos casos, por medición directa de los catastros digitales de La Habana y Madrid. 

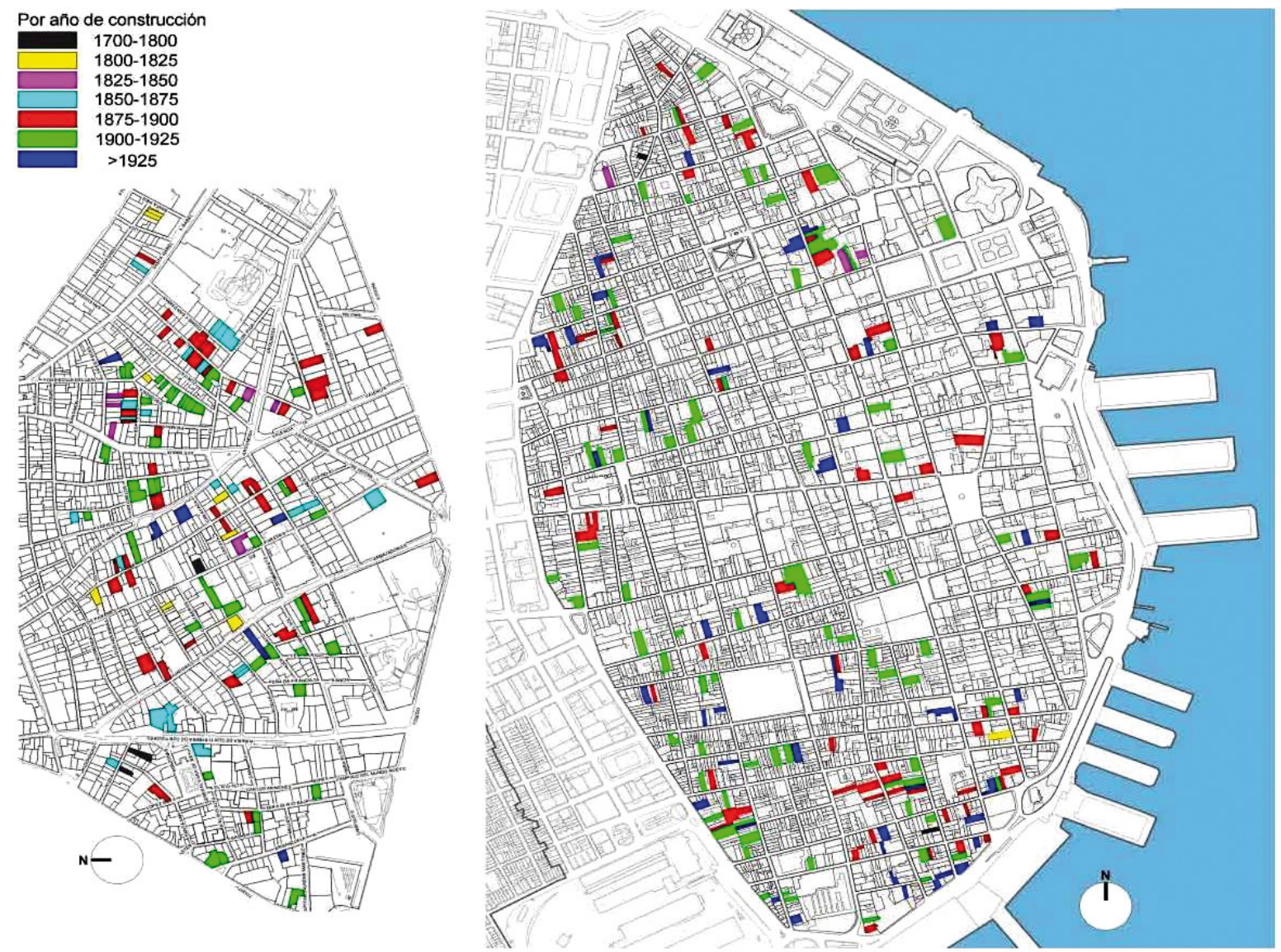

Figura 5. Ámbito de estudio y fecha de construcción de las corralas de Embajadores y ciudadelas de La Habana Vieja.

Es importante reseñar que el 78,8 \% de las ciudadelas y el $65,5 \%$ de las corralas, están edificadas en solares de menos de $400 \mathrm{~m}^{2}$ y de estas más de una tercera parte lo están en solares menores de $200 \mathrm{~m}^{2}$.

En la Figura 7, se muestran los datos relativos al grado de ocupación, entendiendo como tal la diferencia entre la superficie del solar y la de los patios (de corredor y de luces). El $74,1 \%$ de las ciudadelas, y el 65,2 \% de las corralas presentan grados de ocupación por encima del $80 \%$, y un 14,4 \% y un $20 \%$ respectivamente por encima del $90 \%$. Es evidente la elevada densificación, lo que conlleva (con un valor medio de ocupación del 83,65 \% en el caso de las ciudadelas y del $81,80 \%$ en el de las corralas), un importante nivel de infravivienda debida tanto a la baja superficie de las viviendas como a una insuficiente ventilación e iluminación (Tabla 4).

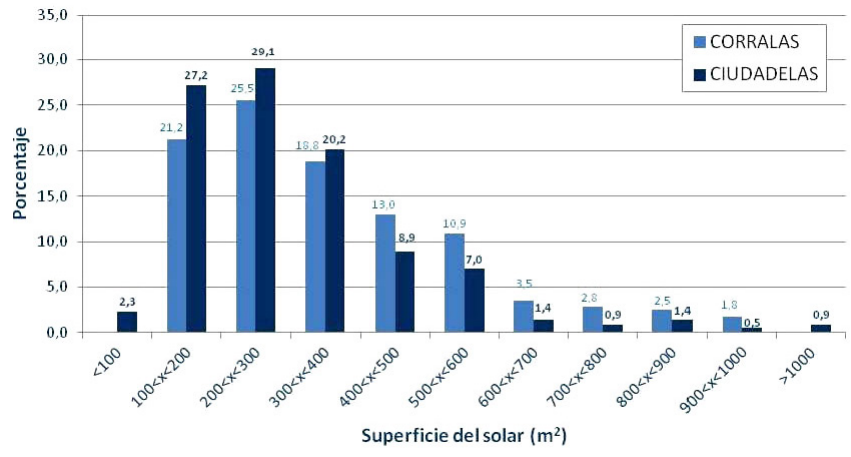

Figura 6. Porcentaje de edificios según superficie del solar.
Estos valores tan altos son consecuencia directa del proceso de implantación de esta tipología edificatoria en la trama urbana, mediante la colmatación de los espacios interiores de las manzanas para obtener una mayor superficie destinada a viviendas.

Los datos de la Figura 8 muestran la superficie edificada sobre rasante (en La Habana Vieja no existen sótanos y en el barrio de embajadores hay muy pocos casos).

En la Figura 9 se muestran los datos referentes al número de plantas. Mientras que las ciudadelas suelen presentar el mismo o menor número de plantas en el patio que en la fachada a la calle, en las corralas por imposiciones legales (1) sucede lo contrario, sobre todo en los edificios de 2, 3 o 4 plantas a calle.

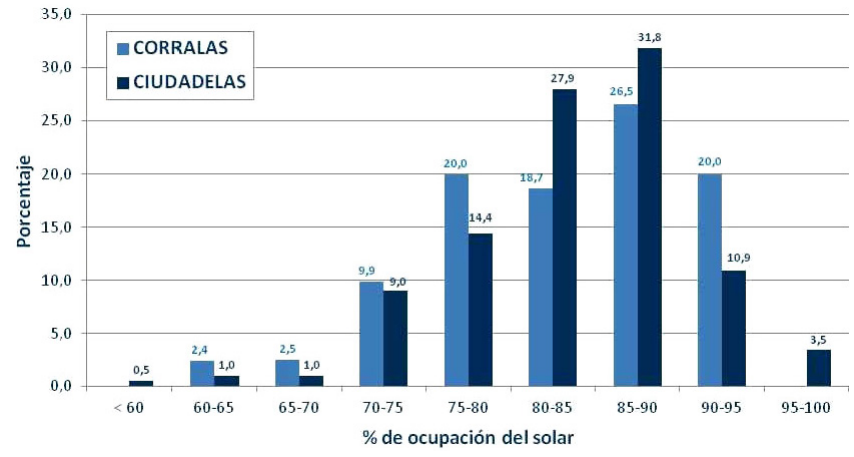

Figura 7. Porcentaje de edificios según ocupación del solar. 
Tabla 4. Datos sobre Infraviviendas por superficie y por ventilación/iluminación.

\begin{tabular}{|c|c|c|c|c|c|}
\hline \multirow{2}{*}{$\begin{array}{l}\text { Ciudadelas: } \\
\text { viviendas }\end{array}$} & \multirow{2}{*}{$\begin{array}{l}\text { No total } \\
\text { viviendas }\end{array}$} & \multicolumn{2}{|c|}{ Infraviv. $x$ superficie } & \multicolumn{2}{|c|}{ Infraviv. $x$ vent/ilum. } \\
\hline & & $\begin{array}{l}\text { Porcentaje } \\
\text { s/ total viv. }\end{array}$ & $\begin{array}{c}\text { Sup. Media } \\
\text { infraviv. }\end{array}$ & $\begin{array}{l}\text { Porcentaje } \\
\text { s/ total viv. }\end{array}$ & $\begin{array}{l}\text { Sup. Media } \\
\text { infraviv. }\end{array}$ \\
\hline Interiores & 2.395 & $73,5 \%$ & $18,1 \mathrm{~m}^{2}$ & $21,3 \%$ & $22,6 \mathrm{~m}^{2}$ \\
\hline Exteriores & 843 & $31,8 \%$ & $19,8 \mathrm{~m}^{2}$ & $6,5 \%$ & $41,9 \mathrm{~m}^{2}$ \\
\hline Totales: & 3.238 & $62,7 \%$ & $18,3 \mathrm{~m}^{2}$ & $17,3 \%$ & $24,8 \mathrm{~m}^{2}$ \\
\hline
\end{tabular}

\begin{tabular}{|c|c|c|c|c|c|}
\hline \multirow{2}{*}{$\begin{array}{l}\text { Corralas: } \\
\text { viviendas }\end{array}$} & \multirow{2}{*}{$\begin{array}{l}N^{\circ} \text { total } \\
\text { viviendas }\end{array}$} & \multicolumn{2}{|c|}{ Infraviv. $x$ superficie } & \multicolumn{2}{|c|}{ Infraviv. $x$ vent/ilum. } \\
\hline & & $\begin{array}{l}\text { Porcentaje } \\
\text { s/ total viv. }\end{array}$ & $\begin{array}{l}\text { Sup. Media } \\
\text { infraviv. }\end{array}$ & $\begin{array}{l}\text { Porcentaje } \\
\text { s/ total viv. }\end{array}$ & $\begin{array}{l}\text { Sup. Media } \\
\text { infraviv. }\end{array}$ \\
\hline Interiores & 1.602 & $52,0 \%$ & $21,3 \mathrm{~m}^{2}$ & $18,8 \%$ & $22,6 \mathrm{~m}^{2}$ \\
\hline Exteriores & 797 & $12,8 \%$ & $22,7 \mathrm{~m}^{2}$ & $8,9 \%$ & $41,9 \mathrm{~m}^{2}$ \\
\hline Totales: & 2.399 & $39,0 \%$ & $21,5 \mathbf{m}^{2}$ & $14,2 \%$ & $24,8 \mathrm{~m}^{2}$ \\
\hline
\end{tabular}

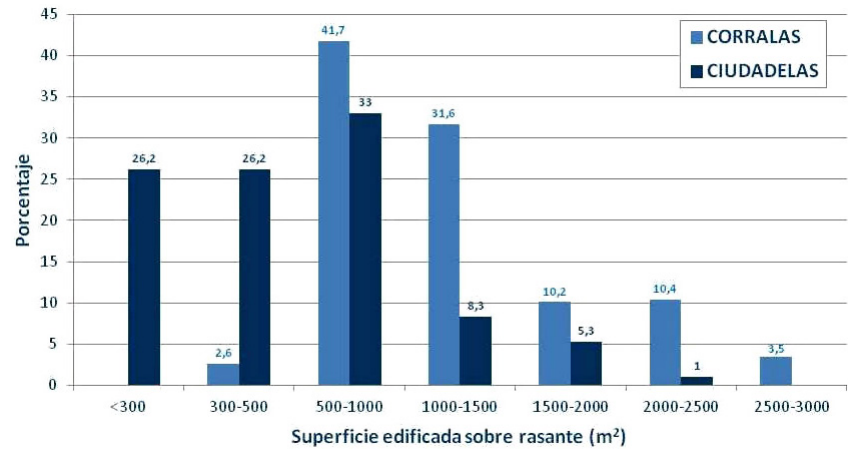

Figura 8. Porcentaje de edificios según superficie edificada sobre rasante.

En general, tanto en las ciudadelas, como en las corralas, cuando se detecta una planta más con fachada a patio, suele ser porque se trata de una edificación agregada con posterioridad, a causa del proceso de densificación del centro histórico.

Estudiando los datos obtenidos también se comprueba que las alturas más frecuentes en las ciudadelas habaneras son de 1, 2 y 3 plantas, mientras que las corralas madrileñas presentan 4 o 5 plantas en la mayoría de los casos, lo que corrobora la causa de la mayor densidad de población en Embajadores.

Estos datos, unidos a los de los altos índices de ocupación de los solares anteriormente indicados, pueden dar una idea del bajo nivel de iluminación de sus viviendas interiores.

La Figura 10 refleja la subdivisión media de los edificios en viviendas exteriores (con toda o parte de su fachada a la calle) e interiores (con toda su fachada al patio de corredor). El que el número de viviendas interiores sea sensiblemente mayor que el de viviendas exteriores (un $74 \%$ en las ciudadelas y un $66 \%$ en las corralas) es consecuencia directa de los datos anteriores de tamaño del solar y ocupación.

\section{Caracterización del patio}

Por último y considerando la caracterización del patio, cada edificio presenta patios de corredor de diversas formas, tamaños y ubicaciones dentro de la parcela que ocupan, representando en su conjunto un amplio repertorio de adaptaciones funcionales.

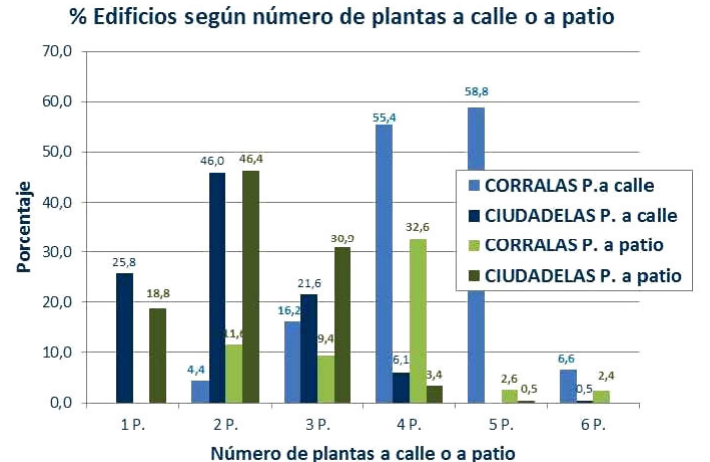

Figura 9. Porcentaje de edificios según el número de plantas a calle y a patio.

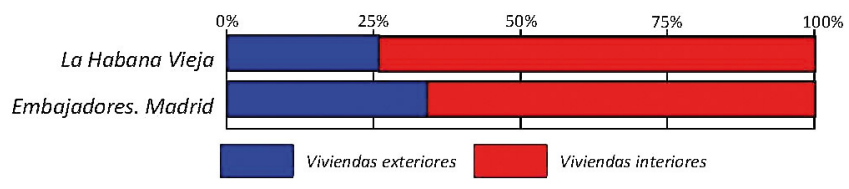

Figura 10. Promedio del número de viviendas exteriores e interiores por edificio.

En la Ficha de Inventario se definieron inicialmente nueve tipos de patios (Tabla 5).

De ellos, sólo el tipo $C$ es un patio totalmente interior, pues el resto se trata de patios que se adosan a una de las medianerías y por lo tanto pueden estar unidos a patios colindantes, formando patios de mayores dimensiones, aunque normalmente estén divididos a nivel de planta baja por una tapia medianera.

Del resultado de la clasificación por tipos de patio de las ciudadelas y corralas inventariadas, recogido en la Tabla 5 , se desprende que solamente los tipos de patio $A, B, C$ y $D$ resultan relevantes, dado que suman el $88,7 \%$ y el $84,3 \%$ del total de las ciudadelas y corralas respectivamente.

Por ello podemos considerar éstas formas de patio como las más representativas de las casas de corredor (Figura 11).

Basándose en el estudio de los datos mostrados en la Tabla 5 , se puede definir como modelo más representativo de patio de corredor al tipo $C$, hecho que queda avalado por el origen 
Tabla 5. Tipos de patios.

\begin{tabular}{|c|c|c|c|c|c|c|c|c|c|c|}
\hline & 든 & 首 & 2 & 2 & & 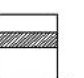 & प्र & पया & - & \\
\hline & A & B & $\mathrm{C}$ & D & E & F & G & $\mathrm{H}$ & I & Otros \\
\hline Ciudadelas & $6,1 \%$ & $10,3 \%$ & $28,6 \%$ & $43,7 \%$ & $0 \%$ & $0 \%$ & $0,5 \%$ & $0,9 \%$ & $1,4 \%$ & $8,5 \%$ \\
\hline Corralas & $4,7 \%$ & $5,8 \%$ & $54,7 \%$ & $29,1 \%$ & o \% & $1,1 \%$ & $0 \%$ & $0 \%$ & $0,2 \%$ & $4,4 \%$ \\
\hline
\end{tabular}

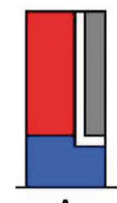

A

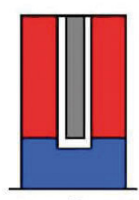

B

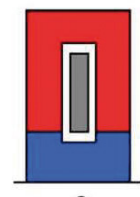

C

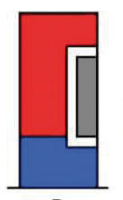

D
Viviendas interiores Viviendas exteriore Patios de corredor

Figura 11. Tipos de patios más representativos.

y evolución de éste tipo de edificios, y por ser la forma que mejor se adapta a la doble función de:

- Patio de iluminación y ventilación.

- Acceso a las viviendas interiores.

Los tipos de patio $B$ y $D$ son meras adaptaciones del tipo C (modelo original) a los distintos solares dependiendo de su tamaño y proporción. El patio tipo $B$ es la adaptación del modelo $C$ a solares con insuficiente fondo como para poder ubicar viviendas en su parte posterior. El patio tipo $D$ es a su vez una adaptación del modelo a solares con insuficiente ancho (o frente de fachada) como para ubicar viviendas en ambos lados del patio.

Existen casos en los que la forma y ubicación del patio no se asemejan a ninguno de los tipos descritos, y por lo tanto se considerarán como casos especiales y se engloban bajo la denominación otros. Normalmente son la consecuencia de geometrías de parcela muy irregulares, o bien de solares en esquina o entre dos calles (Figura 12).

Un 69,67\% de las parcelas habaneras presenta una geometría regular, entendiendo como tal aquellas de planta aproximadamente rectangular, con ángulos de $90^{\circ} \pm 10^{\circ}$ en los vértices. En el caso de los solares del barrio de Embajadores, este resultado es del 51,18 \%. Así, se puede determinar que la geometría de las parcelas de La Habana Vieja es, en la mayoría de los casos, más regular que la de las del barrio madrileño de Embajadores, circunstancia ésta debida a una trama urbana cuasi ortogonal, a diferencia de la trama madrileña formada por las típicas manzanas alargadas de barrios extramuros, con casas de pequeño frente y huerto trasero. $\mathrm{Al}$ fin y al cabo, el trazado de ciudades en ultramar se distinguió, desde el principio, por la adopción de una trama a regla $y$ cordel, tal y como recogen las Leyes de Indias.

\section{CONCLUSIONES}

Pese a que la consolidación completa del barrio de Embajadores se produce en torno a 1800 y que las ciudadelas que aún persisten en La Habana Vieja se edificaron, en su gran mayoría, entre 1825 y 1938, se han encontrado grandes coincidencias en ambos modelos, como:

- Una elevada densificación, lo que conlleva a un importante nivel de infravivienda debida tanto a la baja superficie de las viviendas como a una insuficiente ventilación e iluminación.
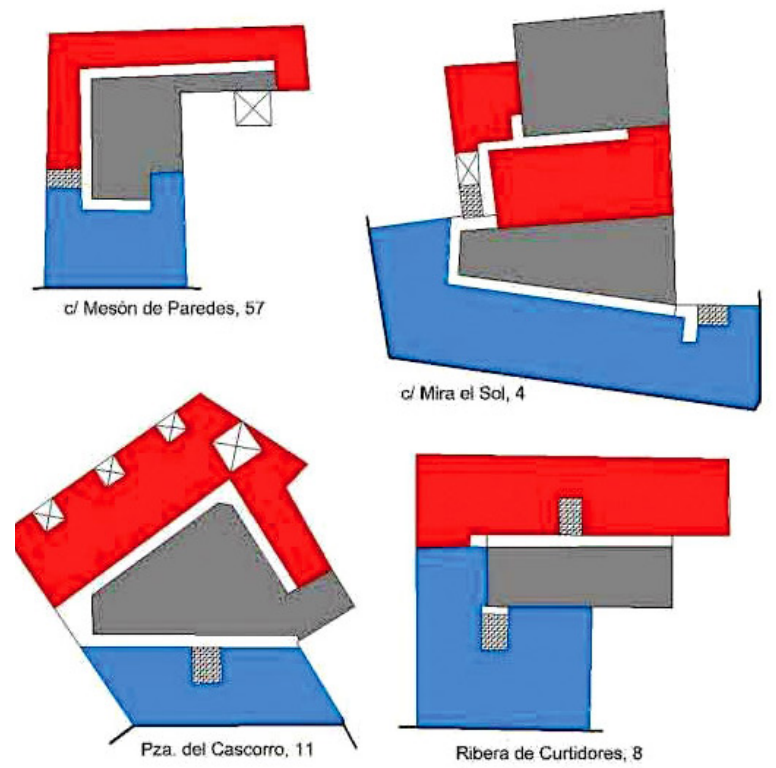

Casas de corredor en Madrid

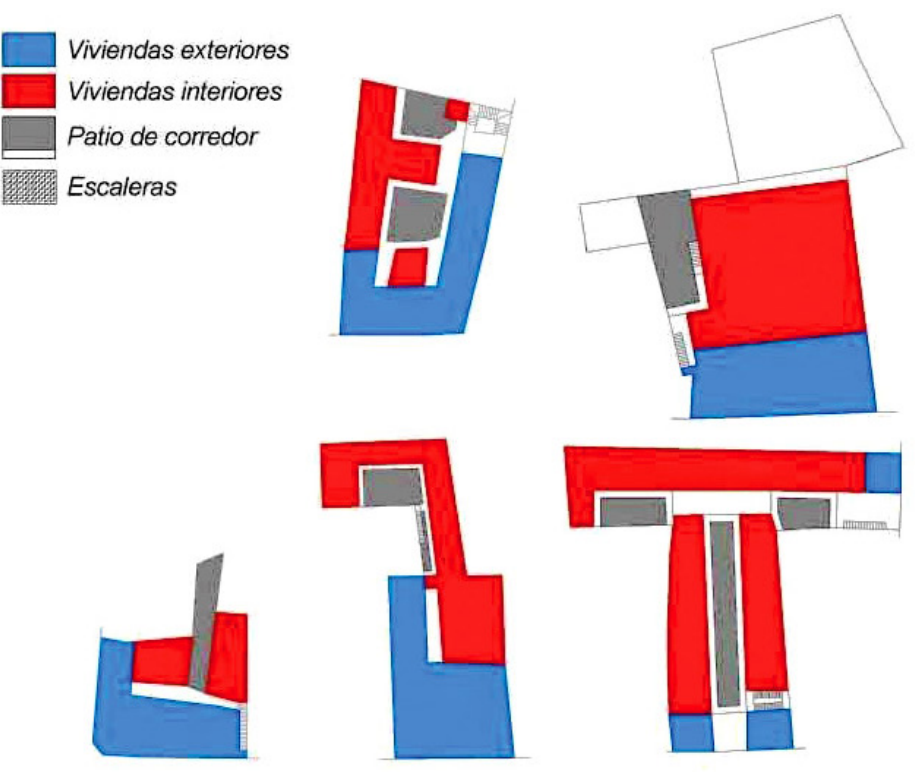

Ciudadelas en La Habana

Figura 12. Ejemplos de ciudadelas con solares de forma irregular. 
- Una cierta homogeneidad en el tamaño y la ocupación media de los solares.

- Un modelo de patio de corredor altamente representativo, y que caracteriza el origen y evolución de éste tipo de edificios, y que permite una doble función:

- Patio de Iluminación y ventilación.

- Acceso a las viviendas interiores.

El modelo original de patio, centrado en la parcela, es el predominante cuando el ancho del solar lo permite, surgiendo como una adaptación el adosado a una medianería, cuando el ancho es insuficiente para poder ubicar viviendas a ambos lados del patio.

En cuanto a las diferencias cabe destacar que la geometría de las parcelas de La Habana Vieja es habitualmente más regu- lar que la geometría de las del barrio madrileño, así como que las alturas más frecuentes en las ciudadelas habaneras son de 1, 2 y 3 plantas, mientras que las corralas madrileñas presentan 4 o 5 plantas en la mayoría de los casos, lo que hace que la densidad de población sea algo más del doble en el caso madrileño que en La Habana Vieja.

En definitiva, se puede concluir que se trata, por tanto, de un modelo tipológico característico de los barrios populares antiguos, y como consecuencia, aplicable tanto a la Habana Vieja como al barrio madrileño de Embajadores y que su presencia es tan alta que resulta imposible explicar la trama urbana sin considerar las ciudadelas o las corralas, fundamentalmente por su papel de «relleno» de las manzanas.

\section{REFERENCIAS}

(1) Santa Cruz Astorqui, J. (2012). Estudio tipológico, constructivo y estructural de las casas de corredor de Madrid (Tesis Doctoral). Madrid: Escuela Universitaria de Arquitectura Técnica - Universidad Politécnica De Madrid.

(2) Ortiz Sanz, J., Rego Sanmartín, T., Cañas Guerrero, I. (2001). La Casa de Corral: Emblema de las construcciones agrarias tradicionales en Castilla León. Valladolid: Junta De Castilla y León - Consejería De Agricultura Y Ganadería.

(3) Piñera Entralgo, L.M. (2007). Ciudadelas, patios, callejones y otras formas similares de vida obrera en Gijón (186o1960). Gijón: Ayuntamiento De Gijón.

(4) Carloni, A. (1984). La mujer en el corral de vecinos sevillano. Revista Etnografía Española, (4): $209-281$.

(5) Fernández Martín, R. (2016). Inventario morfológico de las ciudadelas del casco histórico de La Habana para su caracterización como tipología específica. Estudio comparado con las corralas madrileñas (Tesis Doctoral). Madrid: Escuela Técnica Superior de Edificación - Universidad Politécnica de Madrid.

(6) López Rodríguez, F., Rodríguez Roodríguez, V., Santa Cruz Astorqui, J., Torreño Gómez. I. (2003-2004). Estudio urbanístico y constructivo de las casas de corredor en Madrid. Investigación y documentación de las tipologías edificatorias y constructivas de las denominadas «Casas De Corredor» o «Corralas» en la ciudad de Madrid a partir de una relación de edificios proporcionada por la Empresa Municipal de la Vivienda (EMV). Trabajo de investigación realizado para la EMV y el COAATM. Madrid: Universidad Politécnica de Madrid.

(7) IBM. Software SPSS. http://www-01.ibm.com/software/es/analytics/spss/.

(8) Menéndez García, M. (2006). Tipología de la arquitectura doméstica del centro histórico La Habana Vieja. Su aplicación a los programas de rehabilitación (Tesis Doctoral). Ispjae: Facultad de Arquitectura.

(9) Coca García, O. (2003). La Ciudadela. transformación y desarrollo. Revista Arquitectura y Urbanismo, 24(2).

(10) Zardoya Loureda, M.V. (2003). La Ciudadela Habanera. Antecedentes Históricos. Revista Arquitectura y Urbanismo, 24(2). 